\title{
A Review on - Fast Disintegrating Drug Delivery System
}

\author{
Swapna Mane*, Ritesh Bathe
}

Department of Pharmaceutics, Sahyadri College of pharmacy, Methwade, Sangola, Solapur, Maharashtra, India *Corresponding Author: Swapna mane

\section{ABSTRACT}

Now day's formulation research is breaking barriers of conventional methods. Recently, MDTs have take over an important position in the market by overcoming previously administration problems and contributing to extension of patient life, which have difficulty in swallowing tablets and capsules. Upon introduction into the mouth, these tablets dissolve/ disintegrate in the mouth without additional water for easy administration of pharmaceutical ingredients. These dosage forms are also used to attain instant a higher concentration of drug in body for immediate actions. These are novel dosage forms which dissolve in mouth cavity within a few seconds. This article attempts at discussing ideal properties, advantages, limitation, choice of drug candidates, need of formulation, approaches for preparation of MDTs, Patented technologies on MDTs and Evaluation tests of MDTs.

Keywords : Drug Delivery, Superdisntegranting Agent, Formulation Technology, Patented Technologies, Characterization.

\section{INTRODUCTION}

${ }^{1,2}$ fast disintegrating drug delivery system(FDDTS) are a new generation of formulation which combine the advantages of both liquid and conventional tablet formulation and at the same time offer added advantages over both the traditional dosage forms. They provide the convenience of tablet formulation and allow the ease of swallowing provided by a liquid formulation. FDDS provides much more accurate dosing than the primary alternativeoral liquids. This segment of formulation is espaicially designed for dysphagic, geriatric, pediatric, travelling and psychotic patients who are unable to swallow or refuse to swallow conventional oral formulations. Dysphagia or problem in swallowing is common in all age groups.The FDT is also called as fast melting, fast dispersing orodispersible tablet, rapid dissolve, and rapid melt tablet. The oral route of administration is considered as the most widely accepted route because of its convenience of self medication, compaction, ease of manufacturing, ease of administration, accurate dose, safest and economical route. It is the duty of the health care provider to administer bitter drugs orally with acceptable level of palatability especially with pediatric and geriatric patients. The most evident drawback of the commonly used oral dosage forms like tablets and capsules is swallowing, particularly in case of pediatric and geriatric patients. To fulfill these medical needs, pharmaceutical technologists have developed a novel oral dosage forms known as orally disintegrating tablets (ODTs) or Fast disintegrating tablets (FDTs) or mouth melting tablets(MMTs) or mouth dissolving tablets(MDTs) which disintegrate rapidly in saliva, usually in a matter of seconds, without the need to take water. Drug dissolution and absorption as well as onset of clinical effect and drug bioavailability may be significantly greater than those observed from conventional dosage forms. When such tablets are 
placed in oral cavity, saliva quickly penetrates into the pores to cause rapid tablet disintegrate. Recent market studies indicate that more than half of the patient population prefers FDTs to other dosage forms. Mouth dissolving tablets are formulated mainly by two techniques first use of superdisintegrants like croscarmellose sodium, sodium starch glycolate and crosspovidone. Another method is maximizing pore structure of the tablets by freeze drying and vacuum drying. The bioavailability of some drugs may be increased due to absorption of drugs in oral cavity and also due to pregastric absorption of saliva containing dispersed drugs that pass down into the stomach. Moreover the amount of drug that is subjected to first pass metabolism is reduced as compared to standard tablets.

\section{Salient features of fast dissolving drug delivery system:- $-3,5$}

$>$ Ease of Administration to the patient who cannot swallow, such as the elderly, stroke victims, bedridden patients, patient affected by renal failure and patient who refuse to swallow Pediatric, geriatric \& psychiatric patients.

$>$ No need of water to swallow the dosage form, which is highly convenient feature for patients who Are traveling and do not have immediate access to water.

$>$ Rapid dissolution and absorption of the drug, which will produce quick onset of action.

$>$ Some drugs are absorbed from the mouth, pharynx and esophagus as the saliva passes down into the Stomach. In such cases bioavailability of drug increased.

$>$ Pre-gastric absorption can result in improved bioavailability and as a result of reduced dosage; Improve clinical performance through a reduction of unwanted effects.

$>$ Good mouth feel property helps to change the perception of medication as bitter pill particularly in pediatric patient.
The risk of chocking or suffocation during oral administration of conventional formulation due to Physical obstruction is avoided, thus providing improved safety.

$>$ New business opportunity like product differentiation, product promotion, patent extensions and life cycle management.

$>$ Beneficial in cases such as motion sickness, sudden episodes of allergic attack or coughing, where an ultra rapid onset of action required.

\section{Drug selection criteria for fast dissolving drug delivery:- $-5,6$}

$>$ Drug has no bitter taste; it has good solubility in saliva and water.

$>$ Should be smaller than $50 \mathrm{mg}$.

$>$ Should be low to moderate molecular weight.

$>$ Partially unionized at oral cavity $\mathrm{PH}$.

$>$ Ability to permeate oral mucosal tissue.

$>$ Ability to diffuse and partition into the epithelium of the upper GIT (log $>1$, preferably $>2$ ).

\section{Advantages of fast dissolving drug delivery: $-7,8$}

$>$ No need of water

$>$ Improved patient compliance.

$>$ No need of chewing

$>$ Better taste

$>$ Improved stability

$>$ Suitable for controlled as well as fast release

$>$ Allow high drug loading

$>$ Cost effective.

$>$ Ability to provide advantages of liquid mechanism in the form of solid preparation.

\section{Limitation of fast dissolving drug delivery system: ${ }^{9}$}

$>$ The tablets usually have insufficient mechanical strength. So, careful handling is required.

> The tablets may leave unpleasant taste and/or grittiness in mouth if not formulated properly. 
Drugs with relatively larger doses are difficult to formulate into MDT e.g. antibiotics like

Amoxicillin with adult dose tablet containing about $500 \mathrm{mg}$ of the drug.

Drug with larger doses are difficult to formulate into FDT e.g.rifampin (600) $\mathrm{mg}$, ethambutol (1000) mg.

\section{METHODS AND MATERIAL}

\section{Potential candidate for fast dissolving tablet: 5, 12, 13}

There are no particular limitations as long as it is a substance which is used as a pharmaceutical Active ingredient.

Analgesics and Anti-inflammatory Agents:

Aloxiprin, Auranofin, Azapropazone, Benorylate,

Diflunisal, Etodolac, Fenbufen,

Fenoprofen,Flurbiprofen, Ibuprofen, Indomethacin,

Ketoprofen, Meclofenamic Acid, MefenamicAcid,

Nabumetone, Naproxen, Oxaprozin,

Oxyphenbutazone, Phenylbutazone, Piroxicam,

Sulindac.

Anthelmintics:

Albendazole, Bephenium Hydroxynaphthoate,

Cambendazole, Dichlorophen, Ivermectin,

Mebendazole, Oxarnniquine, Oxfendazole, Oxantel

Embonate, Praziquantel, Pyrantel

Embonate, Thiabendazole.

Anti-Arrhythmic Agents:

Amiodarone, Disopyramide, Flecainide Acetate,

Quinidine Sulphate

Anti-bacterial Agents:

Benethamine Penicillin, Cinoxacin, Ciprofloxacin, Clarithromycin, Clofazimine,

Cloxacillin, Demeclocycline, Doxycycline,

Erythromycin, Ethionamide, Imipenem,

NalidixicAcid, Nitrofurantoin, Rifampicin,

Spiramycin, Sulphabenzamide, Sulphadoxine,

Sulphamerazine, Sulphacetamide, Sulphadiazine,

Sulphafurazole, Sulphamethoxazole,

Sulphapyridine,

\section{Anti-coagulants:}

Dicoumarol, Dipyridamole, Nicoumalone, Phenindione.

\section{Anti-Depressants:}

Amoxapine, Ciclazindol, Maprotiline, Mianserin, Nortriptyline, Trazodone, Trimipramine Maleate. Acetohexamide, Chlorpropamide, Glibenclamide, Gliclazide, Glipizide, Tolazamide, Tolbutamide.

\section{Anti-Hypertensive Agents:}

Amlodipine, Carvedilol, Benidipine, Darodipine, Diltiazem, Diazoxide, Felodipine, Guanabenz

Acetate, Indoramin, Isradipine, Minoxidil,

Nicardipine, Nifedipine, Nimodipine,

Phenoxybenzamine, Prazosin, Reserpine, Terazosin.

\section{Anti-Malarials:}

Amodiaquine, Chloroquine, Chlorproguanil, Halofantrine, Mefloquine, Proguanil.

\section{Anti-Epileptics:}

Beclamide, Carbamazepine, Clonazepam, Ethotoin, Methoin, Methsuximide,

Methylphenobarbitone, Oxcarbazepine,

Paramethadione, Phenacemide, Phenobarbitone, Phenytoin, Phensuximide, Primidone, Sulthiame, Valproic Acid.

\section{Anti-Fungal Agents:}

Amphotericin, Butoconazole Nitrate, Clotrimazole, Econazole Nitrate, Fluconazole,

Fiucytosine, Griseofulvin, Itraconazole, Ketoconazole, Miconazole, Natamycin, Nystatin, Sulconazole Nitrate, Terbinafine, Terconazole, Tioconazole, Undecenoic Acid.

Anti-Gout Agents: Allopurinol, Probenecid, and Sulphinpyrazone.

Superdisintigrants used in fast dissolving tablet:-14, 15, 16, 17,18

Mechanism of disintegration by superdisintigrants:-

a) By capillary action

b) By swelling

c) Because of heat of wetting

d) Due to release of gases

e) By enzymatic action

f) Due to disintegrating particle/particle repulsive forces

g) Due to deformation 


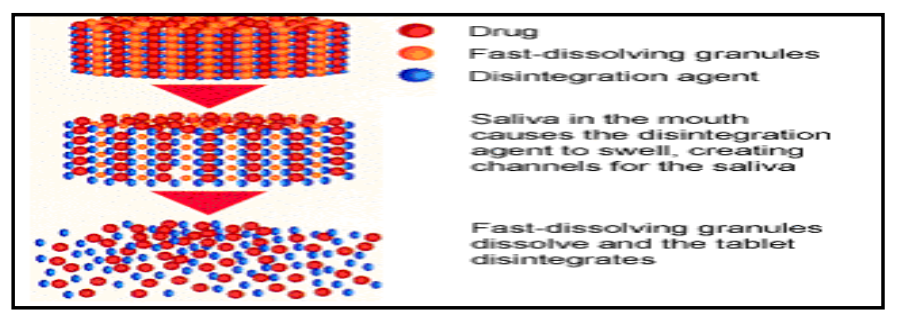

Fig 1.3: Mechanism of Action of Superdisintegrants

\section{a) By capillary action}

Disintegration by capillary action is always the first step. When we put the tablet into suitable aqueous medium, the medium penetrates into the tablet and replaces the air adsorbed on the particles, which weakens the intermolecular bond and breaks the tablet into fine particles. Water uptake by tablet depends upon hydrophilicity of the drug /excipient and on tableting conditions. For these types of disintegrants, maintenance of porous structure and low interfacial tension towards aqueous fluid is necessary which helps in disintegration by creating a hydrophilic network around the drug particles.

\section{b) By swelling}

Perhaps the most widely accepted general mechanism of action for tablet disintegration is swelling Tablets with high porosity show poor disintegration due to lack of adequate swelling force. On the other hand, sufficient swelling force is exerted in the tablet with low porosity. It is worthwhile to note that if the packing fraction is very high, fluid is unable to penetrate in the tablet and disintegration is again slows down.

Wicking
Disintegrant pulls water into the
pores and reduces the physical
bonding forces between particles

Fig 1.4 : Disintegration of Tablet by Wicking and Swelling

\section{c) Because of heat of wetting (air expansion)}

When disintegrants with exothermic properties gets wetted, localized stress is generated due to capillary air expansion, which helps in disintegration of tablet. This explanation, however, is limited to only a few types of disintegrants and cannot describe the action of most modern disintegrating agents.

\section{d) Due to release of gases}

Carbon dioxide released within tablets on wetting due to interaction between bicarbonate and carbonate with citric acid or tartaric acid. The tablet disintegrates due to generation of pressure within the tablet. This effervescent mixture is used when pharmacist needs to formulate very rapidly dispersible tablets or fast disintegrating tablet. As these disintegrants are highly sensitive to small changes in humidity level and temperature, strict control of environment is required durin $g$ manufacturing of the tablets. The effervescent blend is either added immediately prior to compression or can be added into two separate fraction of formulation.

\section{e) By enzymatic reaction}

Here, enzymes present in the body act as disintegrants. These enzymes destroy the binding action of binder and helps in disintegration. Actually due to swelling, pressure exerted in the outer direction or radial direction, it causes tablet to burst or the accelerated absorption of water leading to an enormous increase in the volume of granules to promote disintegration.

\section{f) Due to disintegrating particle/particle repulsive forces}

Another mechanism of disintegration attempts to explain the swelling of tablet made with 'nonswellable' disintegrants. Guyot-Hermann has proposed a particle repulsion theory based on the observation that non-swelling particle also cause disintegration of tablets. The electric repulsive forces between particles are the mechanism of disintegration 
and water is required for it. Researchers found that repulsion is secondary to wicking.

\section{g) Due to deformation}

Hess had proved that during tablet compression, disintegrated particles get deformed and these deformed particles get into their normal structure when they come in contact with aqueous media or water. Occasionally, the swelling capacity of starch was improved when granules were extensively deformed during compression. This increase in size of the deformed particles produces a breakup of the tablet. This may be a mechanism of starch and has only recently begun to be studied.

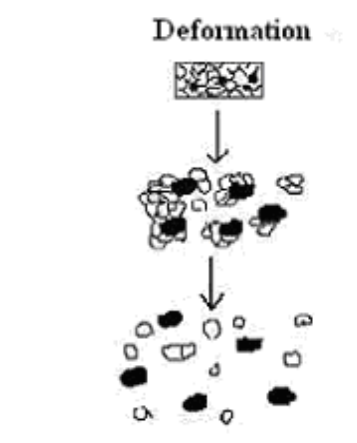

Particles swell to precompression size and break up the matrix

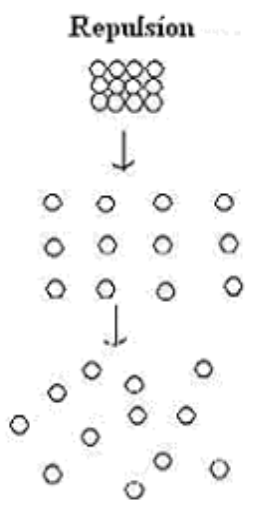

Water is drawn into the pores and particles repel each other due to th resulting electical force
Fig 1.5 : Disintegration by Deformation and Repulsion

Natural Superdisintegrants: -These superdisinegrating agents are natural in origin and are preferred over synthetic substances because they are comparatively cheaper, abundantly available, non-irritating and nontoxic in nature. The natural materials like gums and mucilages have been extensively used in the field of drug delivery for their easy availability, cost effectiveness, Eco friendliness, emollient and nonirritant nature, and non-toxicity, capable of multitude of chemical modifications, potentially degradable and compatible due to natural origin. There are several gums and mucilages are available which have supedisintegrating activity.
Lepidium sativum Mucilage:-

Lepidiumsativum (family: Cruciferae) is known as asaliyo and is widely used as herbal medicine in India. It is widely available in market and has very low cost. Parts used are leaves, root, oil, seeds etc. Seeds contain higher amount of mucilage, dimeric imidazole alkaloids lepidine B, C, D, E and F and two new monomeric imidazole alkaloids semilepidinosidA and B Mucilage of Lepidiums ativumhas various characteristic like binding,disintegrating, gelling.

Plantago Ovata Seed Mucilage (Isapgula):-

Isapghula consists of dried seeds of the plant:-plantago ovata and it contains mucilage which is present in the epidermis of the seeds. The seeds of Plantago ovata were soaked in distilled water for $48 \mathrm{hrs}$ and then boiled for few minutes for complete release of mucilage into water. The material was squeezed through muslin cloth for filtering and separating out the marc. Then, an equal volume of acetone was added to the filtrate so as to precipitate the mucilage. The separated mucilage was dried in oven at temperature less than $60^{\circ} \mathrm{C}$.The mucilage of plantago ovata is a recent innovation for its superdisintegration property when compared with Crospovidone. It shows faster disintegration time than the superdisintegrant, Crosspovidone.

Fenugreek Seed Mucilage: - Trigonella Foenumgraceum, commonly known as Fenugreek, is an herbaceous plant of the leguminous family. It has found wide applications as a food, a food additive, and as a traditional medicine. The leaves and both the ripe and unripe seeds of Trigonella Foenum-graceumare used as vegetables. Fenugreek has been used in treating colic flatulence, dysentery, diarrhoea, dyspepsia with loss of appetite, chronic cough, dropsy, enlargement of liver and spleen, rickets, gout, and diabetes. It is also used as gastro protective, antiurolithiatic, diuretic, antidandruff agent, Antiinflammatory agent and as antioxidant. The seed is stated to be a tonic. It also is used in post-natal care and to increase lactation in nursing mothers. Fenugreek seeds contain a high percentage of 
mucilage (a natural gummy substance present in the coatings of many seeds). Although it does not dissolve in water, mucilage forms a viscous tacky mass when exposed to fluids. Like other mucilage-containing substances, fenugreek seeds swell up and become slick when they are exposed to fluids. The resulting soft mass is not absorbed by the body, but instead passes through the intestine and trigger intestine muscle contraction.

Gum Karaya:-Gum Karaya is a negative colloid and a complex polysaccharide of high molecular weight.On hydrolysis it yields galactose, rhamnose and galacturonic acid. Gum Karaya occurs as apartially acetylated derivative. It is a dried exudation of sterculia Urenstree (Family-Sterculiaceae). Its synonyms are Karaya, sterculia, Indian tragacanth, Bassoratragacanth, kadaya, Kadira, katila. Gum Karaya is compatible with other plant hydrocolloids as well as proteins and carbohydrdrates.

Cassia fistula gum:-

Seeds of Cassia fistula gum obtained from cassia fistula tree. Gum obtained from the seeds of Cassia fistula comprises $\beta$ - $(1 \rightarrow 4)$ linked $d$-mannopyranose units with random distribution of _ $\alpha(1 \rightarrow 6)$ linked $d$ galactopyranose units as side chain having mannose: galactose ratio of 3.0). Carboxymethylation as well as carbamoylethylation of Cassia gum is reported to improve cold water solubility, improve International Journal of Pharmacy and Pharmaceutical Science Research 2012; 2(2) 26-35 viscosity and increase microbial resistance as compared to native gum Therefore, an attempt was made to incorporate calcium or sodium salts of carboxymethylated or carbamoylethylated C. fistula gum as superdisintegrant in the formulation development of FDT.

Locust bean gum: - Is extracted from the endosperm of the seeds of the carob tree Ceretoniasiliqua, which grows in Mediterranean countries. It is also called Carob bean gum. Some other familiar polysaccharides are starch and cellulose, which are made of long chains of the sugar glucose. In locust bean gum, the ratio of mannose to galactose is higher than in guar gum, giving it slightly different properties, and allowing the two gums to interact synergistically so that together they make a thicker gel than either one alone. It shows as a binder and as a disintegrant property at different concentration. Pharmaceutical application of locust bean gum is various novel drug delivery systems. Locust bean gum has been widely used in food industry as a thickening and gelling agent. Locust bean gum has also been reported to have bioadhesive and solubility enhancement properties. There are various reports that Locust bean gum can be used in pharmaceutical and biotechnological purpose.

Guar gum: - Guar gum is a galactomannan, commonly used in cosmetics, food products and in pharmaceutical formulations.Guar gum is mainly consisting of the high molecular weight (approximately 50,000-8,000,000) polysaccharides composed of galactomannans and is obtained from the endosperm of the seed of the guar plant, Cyamopsiste tragonaloba (L) Taub. (SynonymCyamopsispsoraloides). It is used as thickener, stabilizer and emulsifier, and approved in most areas of the world (e.g. EU, USA, Japan, and Australia). Its synonyms are Galactosol; guar flour; jaguar gum; meprogat; meyprodor. It has also been investigated in the preparation of sustained release matrix tablets in the place of cellulose derivatives such as methylcellulose. In pharmaceuticals, guar gum is used in solid-dosage forms as a binder and disintegrant, and in oral and topical products as a suspending, thickening, and stabilizing agent, and also as a controlled-release carrier. Guar gum has also been examined for use in colonic drug delivey.

\section{Synthetic Superdisintegrants}

Cross-linkedpolyvinyl pyrrolidone: (crospovidone, PolyplasdoneXL, XL10) Crospovidone quickly wicks saliva into the tablet to generate the volume expansion and hydrostatic pressures necessary to provide rapid disintegration in the mouth. Unlike 
other superdisintegrants, which rely principally on swelling for disintegration, Crospovidone superdisintegrants use a combination of swelling and wicking. When examined under a scanning electron microscope, crospovidone particles appear granular and highly porous. This unique, porous particle morphology facilitates wicking of liquid into the tablet and particles to generate rapid disintegration. Due to its high crosslink density, crospovidone swells rapidly in water without gelling. Other superdisintegrants have a lower crosslink density and, as a result, form gels when fully hydrated, particularly at the higher use levels in ODT formulations. Swells very little andreturns to original size after compression but act by capillary action.

Sodium Starch Glycolate: (Explotab, Primogel) Sodium starch glycolate is widely used in oral pharmaceuticals as a disintegrant in capsule and tablet formulations. It is recommended to use in tablets prepared by either direct-compression or wetgranulation processes. The recommended concentration in a formulation is $2-8 \%$, with the optimum concentration about $4 \%$ although in many cases $2 \%$ is sufficient. Disintegration occurs by rapid uptake of water followed by rapid and enormous swelling. The disintegrant efficiency of sodium starch glycolate is unimpaired in the presence of hydrophobic excipients, such as lubricants unlike many other disintegrants. Increasing the tablet compression pressure also appears to have no effect on disintegration time. These are modified starches with dramatic disintegrating properties and are available as explotab and primogel which are low substituted carboxy methyl starches. Explotab consisting of granules that absorb water rapidly and swell. The mechanism by which this action takes place involves rapid absorption of water leading to an enormous increase in volume of granules result in rapid and uniform disintegration. The natural predried starches swell in water to the extent of 10-20 percent and the modified starches increase in volume by $200-300$ percent in water.
Modified Cellulose (croscarmellose sodium, Ac-DiSol)

Croscarmellose sodium is described as a cross-linked polymer of carboxy methyl cellulose (CMC). This polymer is different in synthesis and structure as compare to Sodium starch glycolate. Most importantly, the degree of substitution using Williamson"s ether synthesis of croscarmellose sodium is higher than that of sodium starch glycolate, and the mechanism of crosslinking is also different. The chemistry of SSG is different that of cross carmellose sodium. Basic Structure of Croscamellose Sodium the carboxymethyl groups themselves are used to cross-link the cellulose chains. For example, the cross-linking in Primogel are phosphate ester rather than carboxyl ester links as compare to Cross carmellose sodium .Croscarmellose sodium at concentrations up to $5 \% \mathrm{w} / \mathrm{w}$ may be used as a tablet disintegrant, although normally $2 \% \mathrm{w} / \mathrm{w}$ is used in tablets prepared by direct compression and 3\% w/w in tablets prepared by a wet-granulation process.

Resins: - Resins although in soluble, have great affinity for water and hence, act as disintegrant. Moreover, because of their smaller particle size the rate of swelling is high making them superdisintegrant. Like conventional disintegrant, they don't lump but additionally impart strength to the tablets. The use of ion exchange resins into drug delivery systems have been encouraged because of their physico-chemical stability, inert nature, uniform size, spherical shape assisting coating and equilibrium driven reproducible drug release in ionic environment. Ion exchange resins are insoluble polymers that contain acidic or basic functional groups and have the ability to exchange counter-ions within aqueous solutions surrounding them. Drugs, molecules attached to the resins are released by appropriate charged ions in the gastrointestinal tract, followed by diffusion of free drug molecules out of the resins.

\section{Technologies Involved:}




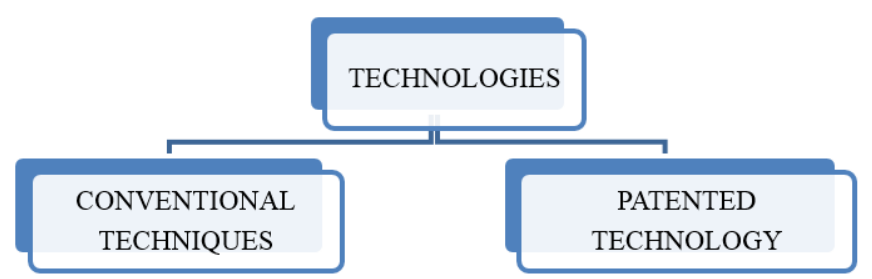

Conventional technique: ${ }^{9,20,21,26,28}$

- Sublimation

- Freeze drying or Lyophilization

- Moulding

- Spray drying

- Direct compression

- Mass extrusion

- Cotton candy process

\section{Sublimation techniques:}

Inert solid ingredients (ex. urea, urethane, camphor, ammonium carbonate, naphthalene) are added to other tablet excipients and the blend is compressed into tablet. Removal of volatile substance by sublimation generated a porous structure. The tablets were dissolving in less than 20 sec. and exhibit sufficient mechanical strength. Conventional compressed tablets that consist highly water-soluble ingredients frequently fall to dissolve rapidly because of low porosity of the matrix. Hence to generate matrix volatile ingredients are used that are later subjected to a process of sublimation. The Heinemann and Rothe, Roser and Blair, Knitsch et.al inert solid ingredients that displayed high volatility (e.g., ammonium bicarbonate, ammonium carbonate, benzoic acid, camphor, hexa methonium tetramine, urea, phthalic anhydride, urethane and naphthalene) were compressed along with other excipients into a tablet. The volatile substance was then removed by sublimation, leaving behind a porous matrix.

\section{Freeze - drying (Lyophilisation technologies):}

In this process water is sublimed from the product after it is frozen. Lyophilisation is a pharmaceutical technology which allows drying of heat sensitive drugs and biological at low temperature under conditions that allow removal of water by sublimation. Lyophilisation results in preparations which have very high specific surface area with a highly porous, which dissolve fastly and show increased absorption and bioavailability. R.P. Scherer patented zydis technology by employing freeze drying process for the preparation of mouth dissolving tablet. On the basis of Gregory et al. Seager formation, process technology \& bioavailability of fast dissolving tablets prepared by using zydis technology.

\section{Molding method:}

Moulded tablets are prepared by using water-soluble ingredients so that the tablet dissolve or disintegrate rapidly and completely. Powder is moistened with the help of hydro alcoholic solvent and then moulded into tablets under pressure less than the conventional form. The tablet possesses porous structures, which provide easy dissolution. Adding sucrose, acacia or PVP k30 may increase the mechanical strength of the tablet, which provides easy dissolution. Adding sucrose, acacia or PVP k30 may increase the mechanical strength of the tablet.

\section{Spray drying technique:}

Spray drying can produce highly porous and fine powders that dissolve rapidly. The formulations are included by hydrolyzed and non hydrolyzed mannitol as bulking agent, gelatin as supporting agents, sodium starch glycolate or croscarmellose sodium as disintegrating agent and an acidic material (e.g. citric acid) and/ or alkali material (e.g. sodium bicarbonate) to improve disintegration \& dissolution.

\section{Direct Compression:-}

It is the easiest way to formulation of tablets. Conventional equipment, easily available excipients and a limited number of processing steps are involved in direct compression. High doses can be accommodated and final weight of tablet can easily exceed that of other production methods.The disintegration and solubilisation of directly compressed tablets depends on single or combined action of disintegrates, effervescent agents and water soluble excipients used. Breakage of tablet edges during handling and tablet crack during the opening of blister alveolus. To essential a high disintegration 
rate, choice of suitable type and an optimal quantity of disintegrate is important. Other components such as water soluble excipients or effervescent agents can promote improved dissolution or disintegration properties. But the main problem of using effervescent excipients is that they are highly hygroscopic in nature.

\section{Mass Extrusion:}

This involves softening the active blend using the solvent mixture of water-soluble polyethylene glycol and methanol and subsequent removal of softened mass through the syringe to get a cylinder of the product into even segments using heated blade to form tablet.

\section{Cotton candy process:}

This process is so named as it utilizes a unique spinning mechanism to produce floss-like crystalline structure, which mimic cotton candy. Cotton candy process 12 involves formation of matrix of polysaccharides or saccharides by simultaneous action of flash melting and spinning. The matrix formed is partially re-crystallized to have improved flow properties and compressibility. This candy floss matrix is then milled and blended with active ingredients and excipients and subsequently compressed to MDT.

\section{Technologies for Fast Dissolving Tablets: 9, 23, 25, 27, 28}

- Durasolv Technology

- Zydis Technology

- Flash Dose Technology

- Orasolv Technology

- Wowtab Technology

- Ceform technology

- Flashtab Technology

- Frosta technology

- Pharmaburst technology

- Lyoc technology
Durasolv technology:-This technology is patented by CIMA labs. The tablets produced by this technology utilize the conventional tableting equipment. Tablets in this are formulated by using drug, nondirect compression fillers and lubricants. Nondirect compressible fillers are dextrose, mannitol, sorbitol, lactose and sucrose, which have advantage of quick dissolution and avoid gritty texture, which is generally present in direct compressible sugars. The tablets obtained are strong and can be packed in conventional packing into bottles and blisters.

Zydis technology:-Zydis technology is the first mouth dissolving dosage form in the market. It is a unique freeze dried tablet in which the active drug is incorporated in a water soluble matrix, which is then transformed in to blister pockets and freeze dried to remove water by sublimation. When zydis units are put into the mouth, the freeze dried structure disintegrates instantaneously and does not require water to aid swallowing. The zydis matrix is composed of many material designed to achieve a number of objectives. Polymers such as gelatin, dextran or alginates are added to impart strength during handling. These form a glossy and amorphous structure. Mannitol or sorbitol is added to impart crystallinity, elegance and hardness. Various gums may be added prevent sedimentation of dispersed drug particles. Water is used as a medium to ensure the formation of a porous dosage form. Collapse protectants like glycine may be used to prevent shrinkage of dosage form during freeze drying and long term storage. If necessary, suspending agents and $\mathrm{pH}$ adjusting agents may be used. Preservatives may also be added to prevent microbial growth. Zydis products are packed in blister packs to protect the formulation from environmental moisture.

Flash tab technology:-Prographarm labs have a patent over this technology. In this technology, microgranules of the taste masked active drug are used. These may be prepared by using conventional techniques like coacervation, microencapsulation and 
extrusion - spheronisation. All these processes utilize conventional tableting technology. These taste masked micro crystals of active drug, disintegrating agent, a swelling agent and other excipients like soluble diluents etc are compressed to form a multiparticulate tablet that disintegrates rapidly.

Orasolv technology:-CIMA labs have developed orasolv technology. In this system active medicament is taste masked. It also contains effervescent disintegrating agent. The evolution of carbon dioxide from the tablet produces fizzing sensation, which is a positive organoleptic property. Concentration of effervescent mixture usually employed is $20-25 \%$ of tablet weight. Tablets are made by direct compression technique at low compression force in order to minimize oral dissolution time. Conventional blenders and tablet machine is used to produce the tablets. The tablets produced are soft and friable.

Wow tab technology:-Wow tab technology is patented by yamanouchi pharmaceutical company wow means "Without water". In this process, combination of low mouldability saccharrides and high mouldability saccharides is used to obtain a rapidly melting strong tablet. The active ingredient is mixed with a low mouldability saccharide and granulated with a high mouldability saccharide and compressed into tablet.

Ceform technology:-In ceform technology microspheres containing ceform active drug ingredient are prepared. The essence of ceform microsphere manufacturing process involves placing a dry powder, containing substantially pure drug material or a special blend of drug materials plus other pharmaceutical compounds, and excipients into a precision engineered and rapidly spinning machine. The centrifugal force of the rotating head of ceform machine throws the dry drug blend at high speed through small, heated openings. The carefully controlled temperature of the resultant microbrust of heat liquefies the drug blend to form a sphere without adversely affecting drug stability. The microspheres are then blended and/ or compressed into the preselected oral delivery dosage format. The ability to simultaneously process both drug and excipients generates a unique microenvironment in which materials can be incorporated into the microspheres that can alter the characteristics of the drug substance, such as enhancing solubility and stability. The microspheres can be incorporated into a wide range of fast dissolving tablets such as flash dose, EZ chew, spoon dose as well as conventional tablets.

Frosta technology:-This technology is patented by Akina. It utilizes the concept of formulating plastic granules and compressing at low pressure to produce strong tablets with high porosity. Plastic granules composed of porous and plastic material, water penetration enhancer and binder. The process involves usually mixing the porous plastic material with water penetration enhancer and followed by granulating with binder. The tablets obtained have excellent hardness and rapid disintegration time ranging from 15 to 30 s depending on size of tablet.

Pharmaburst technology:-SPI Pharma, New Castle, patents this technology. It utilizes the coprocessed excipients to develop FDT's which dissolves within 30 - 40s. This technology involves dry blending of drug, flavour and lubricant followed by compression into tablets. Tablets obtained have sufficient strength so they can be packed in blister packs and bottles.

Lyoc technology:-Lyoc technology is pantented by Pharmalyoc. Oil in water emulsion is prepared and placed directly into blister cavities followed by freeze drying. Non-homogeneity during freeze drying is avoided by incorporating inert filler to increase the viscosity finally the sedimentation. High proportion of filler reduces porosity of tablets due to which disintegration is lowered. 


\section{RESULTS AND DISCUSSION}

Evaluation of fast disintegrating tablets: ${ }^{11,21,25}$

a. Tablet thickness: Tablet thickness is an important characteristic in reproducing appearance and also in counting by using filling equipment. Some filling equipment utilizes the uniform thickness of the tablets as a counting mechanism. Thickness was recorded using micrometer.

b. Hardness (Crushing strength): Tablet hardness is measured with hardness testers like Monsanto. A tablet is placed in the hardness tester and load required to crush the tablet is measured. The hardness of MDTs is generally kept lower than conventional tablets as increased hardness delays the disintegration of the tablet. A good compromise between mechanical strength and disintegration time is achieved for a satisfactory mouth dissolving formulation.

c. Friability: Ten tablets were weighed and placed in a Roche friabilator and it is rotated for $4 \mathrm{~min}$. at $25 \mathrm{rpm}$. The tablets were taken out, dedusted and reweighed. The standard limit of friability is $0.5-1 \%$.

d. Weight variation: Randomly, twenty tablets were selected after compression and the mean weight was determined. None of tablets deviated from the average weight by more than \pm 10.5 (USPXX).8.

e. Drug content: Twenty tablets were weighed and powdered. An amount of powder equivalent to $150 \mathrm{mg}$ of promethazine hydrochloride was dissolved in $100 \mathrm{ml}$ of $\mathrm{pH} 7.4$ phosphate buffer, filtered, diluted suitably and analyzed for drug content at $249 \mathrm{~nm}$ using UV-Visible spectrophotometer.

f. Wetting time and water absorption ratio: Wetting time of dosage form is related to with the contact angle. Wetting time of the MDT is another important parameter, which needs to be assessed to give an insight into the disintegration properties of the tablet. Lower wetting time implies a quicker disintegration of the tablet. Five circular tissue papers of $10 \mathrm{~cm}$ diameter are placed in a petri dish. Ten milliliters of water soluble dye solution is added to petri dish. A tablet is carefully placed on the surface of the tissue paper. The time required for water to reach upper surface of the tablet is noted as the wetting time. For measuring water absorption ration the weight of the tablet before keeping in the petri dish noted $(\mathrm{Wb})$. The wetted tablet from petri dish is taken and reweighed (Wa). The water absorption ratio, $\mathrm{R}$ can be the determined according to the following equation. $\mathrm{R}=100$ (Wa-Wb) / Wb 6 .

g. In vivo disintegration time: In vivo disintegration time is determined using a panel of healthy human volunteers. The DT noted by the volunteers by placing the tablet in mouth.

h. Dissolution test: The dissolution study is perfortmed by use of USP 2 (paddle speed of 50 rpm) apparatus which is most suitable for MDTs. The USP 1 (basket) apparatus may have certain application for such tablets but is used less frequently due to specific physical properties of tablets.

i. Stability study: The mouth dissolving tablets are packed in suitable packaging and stored under the following conditions for a period as prescribed by ICH guidelines for accelerated studies. (i) $40 \pm 1{ }^{\circ} \mathrm{C}$ (ii) $50 \pm 1^{\circ} \mathrm{C}$ (iii) $37 \pm 1{ }^{\circ} \mathrm{C}$ and $\mathrm{RH} 75 \% \pm 5 \%$ The tablets were withdrawn after 15 days and analyzed for physical characterization (Hardness, Friability, Disintegrations, and Dissolution etc.) and drug content. Accelerated stability data are plotting according Arrhenius equation to determine the shelf life at $25^{\circ} \mathrm{C}$.

\section{CONCLUSION}

Fast dissolving tablets are innovative dosage forms developed and specially designed to overcome some 
of the problems that seen in conventional solid dosage form i.e. difficulty in swallowing of the tablet in geriatric and pediatric patients. Fast dissolving tablets are designed to dissolve or disintegrate quickly in the saliva generally within less than 60 seconds (range of 5-60 seconds). Fast dissolving tablets have better patient compliance and acceptance may improve biopharmaceutical properties, bioavailability improved efficacy, convenience, and better safety compared with conventional oral dosage forms. The popularity of FDTs has increased fabulously over the last decade. FDTs need to be formulated for psychotic patients, bedridden, geriatric, pediatric patients, for those patients who may not have easy access to water, patients who are busy in traveling. Hence, in future patient demand and the availability of various technologies have increased the market share of fast dissolving tablets, which in turn prolongs the patient life and expect technology to be more popular.

\section{REFERENCES}

[1]. Baviskar Dheeraj, Jain Dinesh, Novel Drug Delivery System, second Edition, Nirali Prakashan, 2015, 5.4-5.5.

[2]. Ujjwal Nautiyal, Satinderjeet Singh, Fast dissolving tablet A Novel Boon, A Review, Journal of Pharmaceutical, Chemical and Biological Sci, 2014; 2(1), 25-26.

[3]. Bhupendra G Prajapati, Nayan Ratnakar, A Review on recent patent on fast dissolving drug delivery, Int Journal of pharma Tech research, vol-1, 790-780.

[4]. Gupta Dilip kumar, Bajpai Minakshi, fast mouth dissolving and disintegrating tablet, $\mathrm{A}$ review, Int journal of research and devlopement in pharmacy and life science, 2014; vol.3, 949-958.

[5]. Y.A. Choudhary, Soumya.M, A review on fast dissolving drug delivery system-A pioneering drug delivery technology, Bulletin of environment and life science, 2012; 1(12), 820.

[6]. Erande kumar, Joshi Bhagyashree, Mouth dissolving tablet $-\mathrm{A}$ comprehensive review, Int Journal of Pharma and research, 2013;2(7), 25-41.

[7]. Kushagra Khanna, Gauravi Xavier, Fast dissolving tablet-A Novel Approach, Int. J. Pharm. Res. Allied Sci., 2016; 5(2), 311-322.

[8]. Asija R, A Review on fast dissolving drug delivery system, Int J Res Pharm Sci, 2014; 4(3), 7 - 12.

[9]. Rajesh Roshan Rai, Pavithra Chirra, Venkataramudu Thanda. Fast dissolving tablet: A novel approach to drug delivery-A review; Int journal of preclinical and pharma research, 2012; 3(1), 23-32.

[10]. Ajoy Bera, Ashish Mukhrjee, A detailed study of mouth dissolving drug delivery system, Acta Chimica and Pharmaceutica indica, 2013, 3(1).

[11]. Sehgal Prateek, Gupta Ramdayal, Fast dissolving tablet: A New Venture in drug delivery, Am.J.Pharm Tech Res.2012; 2(4).

[12]. Adchitre vaishali B., Puroshattam R. Patil, Fast dissolving tablet $-\mathrm{A}$ novel approach to drug drug delivery; Indo American journal of pharmaceutical research, 2016;6(4), 50095023

[13]. Chhote lal singh, Neeraj Rajput, A review on fast dissolving tablet; world journal of pharmaceutical sciences, 1573-1581.

[14]. P. S. Mohanachandran, P. G. Sindhumol, Superdisintegrants: An Overview. International Journal of Pharmaceutical Sciences Review and Research, 2011;Vol 6, 105-109.

[15]. Mohit mangal, Sunil Thakral, Superdisintigrants: An updated review. Int journal of pharmacy and pharmaceutical science and research, 2012; 2(2), 26-35. 
[16]. Garima Yadav, Anupriya kapoor, fast dissolving tablet advantages: A Review. Int J of Pha sci and Re, 2012; 3 (3), 728-736.

[17]. Uma Shankar Mishra, Dr.S.K. Prajapati, A review on formulation and evaluation of mouth dissolving tablet, W J Pharm Pharma Sci, 2014; 2(1), 5-26.

[18]. Pooja Arora, Vandana Arora, orodispersible tanblet: A comprehensive review, Int J Res Dev Pharm L Sci, 2013; 2(2), 270-284.

[19]. Shilpi Rawat, superdiaintigrant:An overview, world journal of pharmacy and pharmaceutical science, 2014;3(5), 263-278.

[20]. Bhowmik Debjit, Kant Krishna, Fast Dissolving Tablet: An Overview, Journal of Chemical and Pharmaceutical Research, 2009, 1(1), 163-177.

[21]. Sharma Ritika, Rajput Meenu, fast dissolving drug delivery system- A Review. Int Res J Pharm, 2011; 2(11), 21-29.

[22]. Tripathi. KD. Essential of Medical pharmacology. 6th edition. Jaypee publishers; New Delhi, India. 2008; 539-554.

[23]. Chein Y.W. Novel drug delivery system. 2nd edition. New York; Marcel Dekker Inc; 1992; 50:139-40.

[24]. Ghadge SJ, Keskar SR, Oral disintegrating tablets: An Overview. Int J Universal Pharmacy and Life Sci, 2011; 1(3), 35-50.

[25]. Patel GJ, Pandya VM, An overview on future trends in oral formulation technologies: Orally disintegrating tablet. J Pharma and Cosmetology, 2011; 1(4), 42.

[26]. Caramella C, Novel methods for disintegrant characterization, Part 1, Pharm. Technology Int, 1990, 2(9), 30 - 37.

[27]. Parakh SR, Gothosakar AV, A Review of Mouth Dissolving Tablet Technologies," Pharm. Technol, 2003; 27(11), 92-100.

[28]. Dinesh Kumar, Ira Sharma and Vipin Sharma, A comprehensive review on Fast dissolving tablet technology ,J App Pharm Sci ,2011;01(05),50-58.
Cite this article as :

Swapna Mane, Ritesh Bathe, "A Review on - Fast Disintegrating Drug Delivery System", International Journal of Scientific Research in Science and Technology (IJSRST), Online ISSN : 2395-602X, Print ISSN : 2395-6011, Volume 6 Issue 1, pp. 291-303, January-February 2019. Available at doi : https://doi.org/10.32628/IJSRST196139 Journal URL : http://ijsrst.com/IJSRST196139 